\title{
CONNECTION BETWEEN DYNAMICALLY DERIVED INITIAL MASS FUNCTION NORMALIZATION AND STELLAR POPULATION PARAMETERS
}

\author{
Richard M. McDermid ${ }^{1,2}$, Michele Cappellari $^{3}$, Katherine Alatalo $^{4}$, Estelle Bayet $^{3}$, Leo Blitz $^{5}$, Maxime Bois $^{6}$, \\ Frédéric Bournaud ${ }^{7}$, Martin Bureau ${ }^{3}$, Alison F. Crocker ${ }^{8}$, Roger L. Davies ${ }^{3}$, Timothy A. Davis ${ }^{9}$, P. T. De Zeeuw $^{9}, 10$, \\ Pierre-Alain Duc ${ }^{7}$, Eric Emsellem ${ }^{9}, 11$, Sadegh Khochfar ${ }^{12}$, Davor Krajnović ${ }^{13}$, Harald Kuntschner ${ }^{9}$, \\ RafFaella Morganti ${ }^{14,15}$, Thorsten NaAB ${ }^{16}$, Tom Oosterloo ${ }^{14,15}$, Marc Sarzi ${ }^{17}$, Nicholas Scott ${ }^{18}$, \\ Paolo Serra ${ }^{14,19}$, Anne-Marie WeiJmans ${ }^{20}$, and Lisa M. Young ${ }^{21,22}$ \\ ${ }^{1}$ Department of Physics and Astronomy, Macquarie University, Sydney NSW 2109, Australia; richard.mcdermid@mq.edu.au \\ ${ }^{2}$ Australian Gemini Office, Australian Astronomical Observatory, P.O. Box 915, Sydney NSW 1670, Australia \\ ${ }^{3}$ Sub-Department of Astrophysics, Department of Physics, University of Oxford, Denys Wilkinson Building, Keble Road, Oxford, OX1 3RH, UK \\ ${ }^{4}$ Infrared Processing and Analysis Center, California Institute of Technology, Pasadena, CA 91125, USA \\ ${ }^{5}$ Department of Astronomy, Campbell Hall, University of California, Berkeley, CA 94720, USA \\ ${ }^{6}$ Observatoire de Paris, LERMA and CNRS, 61 Av. de 1'Observatoire, F-75014 Paris, France \\ ${ }^{7}$ Laboratoire AIM Paris-Saclay, CEA/IRFU/SAp- CNRS-Université Paris Diderot, F-91191 Gif-sur-Yvette Cedex, France \\ ${ }^{8}$ Ritter Astrophysical Observatory, University of Toledo, Toledo, OH 43606, USA \\ ${ }^{9}$ European Southern Observatory, Karl-Schwarzschild-Str. 2, D-85748 Garching, Germany \\ ${ }^{10}$ Sterrewacht Leiden, Leiden University, Postbus 9513, 2300 RA Leiden, The Netherlands \\ ${ }^{11}$ Université Lyon 1, Observatoire de Lyon, Centre de Recherche Astrophysique de Lyon and Ecole Normale Supérieure de Lyon, \\ 9 avenue Charles André, F-69230 Saint-Genis Laval, France \\ ${ }^{12}$ Institute for Astronomy, University of Edinburgh, Royal Observatory, Edinburgh, EH9 3HJ, UK \\ ${ }^{13}$ Leibniz-Institut für Astrophysik Potsdam (AIP), An der Sternwarte 16, D-14482 Potsdam, Germany \\ ${ }^{14}$ Netherlands Institute for Radio Astronomy (ASTRON), Postbus 2, 7990 AA Dwingeloo, The Netherlands \\ ${ }^{15}$ Kapteyn Astronomical Institute, University of Groningen, Postbus 800, 9700 AV Groningen, The Netherlands \\ ${ }_{16}$ Max-Planck-Institut für Astrophysik, Karl-Schwarzschild-Str. 1, D-85741 Garching, Germany \\ ${ }^{17}$ Centre for Astrophysics Research, University of Hertfordshire, Hatfield, Herts AL1 9AB, UK \\ ${ }^{18}$ Sydney Institute for Astronomy (SIfA), School of Physics, The University of Sydney, NSW 2006, Australia \\ ${ }^{19}$ CSIRO Astronomy \& Space Science, P.O. Box 76, Epping, NSW 1710, Australia \\ ${ }^{20}$ School of Physics and Astronomy, University of St Andrews, North Haugh, St Andrews KY16 9SS, UK \\ ${ }^{21}$ Physics Department, New Mexico Institute of Mining and Technology, Socorro, NM 87801, USA \\ 22 Academia Sinica Institute of Astronomy \& Astrophysics, P.O. Box 23-141, Taipei 10617, Taiwan, R.O.C. \\ Received 2014 June 10; accepted 2014 August 10; published 2014 August 27
}

\begin{abstract}
We report on empirical trends between the dynamically determined stellar initial mass function (IMF) and stellar population properties for a complete, volume-limited sample of 260 early-type galaxies from the ATLAS ${ }^{3 \mathrm{D}}$ project. We study trends between our dynamically derived IMF normalization $\alpha_{\mathrm{dyn}} \equiv(M / L)_{\text {stars }} /(M / L)_{\text {Salp }}$ and absorption line strengths, and interpret these via single stellar population-equivalent ages, abundance ratios (measured as $[\alpha / \mathrm{Fe}])$, and total metallicity, $[\mathrm{Z} / \mathrm{H}]$. We find that old and alpha-enhanced galaxies tend to have on average heavier (Salpeter-like) mass normalization of the IMF, but stellar population does not appear to be a good predictor of the IMF, with a large range of $\alpha_{\mathrm{dyn}}$ at a given population parameter. As a result, we find weak $\alpha_{\mathrm{dyn}}-[\alpha / \mathrm{Fe}]$ and $\alpha_{\mathrm{dyn}}-$ Age correlations and no significant $\alpha_{\mathrm{dyn}}-[\mathrm{Z} / \mathrm{H}]$ correlation. The observed trends appear significantly weaker than those reported in studies that measure the IMF normalization via the low-mass star demographics inferred through stellar spectral analysis.
\end{abstract}

Key words: galaxies: abundances - galaxies: elliptical and lenticular, cD - galaxies: kinematics and dynamics galaxies: stellar content

Online-only material: color figures

\section{INTRODUCTION}

The stellar initial mass function (IMF) of massive galaxies has been the focus of much attention recently, triggered by findings that suggest a non-universal and systematically varying form of the IMF among galaxies in the current day universe (van Dokkum \& Conroy 2010; Cappellari et al. 2012). These findings differ from studies of resolved stellar systems in and near the Milky Way, which indicate that the IMF has a universal form (Kroupa 2002; Bastian et al. 2010). Strong evidence for a non-universal IMF has come from the application of various independent techniques, including gravitational lensing (Auger et al. 2010), stellar dynamical modeling (Cappellari et al. 2012, 2013a; Tortora et al. 2013; Conroy et al. 2013), and spectral synthesis (van Dokkum \& Conroy 2010, 2011; Spiniello et al.
2012, 2014; La Barbera et al. 2013; Ferreras et al. 2013). With few exceptions (e.g., Smith et al. 2012; Smith \& Lucey 2013; Peacock et al. 2014), such studies so far agree that, in general, galaxies with higher velocity dispersion require a "heavy" form of the IMF in order to account fully for the measured total mass-to-light ratio. While this general picture is one of qualitative agreement, there is ongoing debate as to what key parameters drive the IMF variations. In particular, Smith (2014) find a notable discrepancy between methods on a galaxy-bygalaxy basis, and conclude that the dynamically derived IMF normalization for 34 objects in Atlas3D does not correlate with $[\mathrm{Mg} / \mathrm{Fe}]$ after controlling for velocity dispersion.

In this Letter, we report on how the stellar population properties of the complete ATLAS ${ }^{3 \mathrm{D}}$ sample are related to the dynamically derived IMF normalization. This expands 
the sample of 34 objects of Smith (2014) to a total of 212 galaxies, studying age, metallicity, and $[\alpha / \mathrm{Fe}]$, and spanning a significantly larger range of stellar population parameter values.

\section{OBSERVATIONS AND DERIVED QUANTITIES}

All observations come from the ATLAS ${ }^{3 D}$ Survey (Cappellari et al. 2011), and comprise optical integral-field spectroscopy covering half the stellar light, on average (i.e., one effective radius, $R_{e}$ ). The spectral data cubes were spatially integrated to form an effective aperture corresponding to a radius of oneeighth of an effective radius, $R_{e} / 8$. Such an aperture is similar to those used in other (generally long-slit or single-fiber spectrograph) studies in the literature, and allows our results to be more directly compared to them. The single stellar population (SSP) models of Schiavon (2007) were used to measure the SSP-equivalent age, metallicity $[\mathrm{Z} / \mathrm{H}]$, and alpha-element abundance $[\alpha / \mathrm{Fe}]$ (with IMF fixed to that of a unimodal power law of the form: $\zeta(m) \propto m^{-2.35}$, Salpeter 1955) using the chi-squared approach detailed in McDermid et al. (2006), finding the model from an interpolated grid which simultaneously best approximates our measured $\mathrm{H} \beta, \mathrm{Fe} 5015$ and $\mathrm{Mg} b$ line indices on the Lick/IDS system (Worthey \& Ottaviani 1997). A full presentation of the stellar populations for the ATLAS ${ }^{3 \mathrm{D}}$ Survey is given in McDermid et al. (2014).

Stellar kinematics were measured from our integral-field spectroscopy using the pixel fitting code pPXF (Cappellari \& Emsellem 2004), and the resulting maps of stellar line-of-sight mean velocity and velocity dispersion were fitted using general anisotropic Jeans models (Cappellari 2008), in conjunction with multi-Gaussian mass models (Emsellem et al. 1994; Cappellari 2002) from Sloan Digital Sky Survey imaging, which are reported in Scott et al. (2013). The stellar mass-to-light ratios $(M / L)_{\text {stars }}$ we use here were derived accounting for a standard dark matter halo (Navarro et al. 1996), and correspond to models "B" as described in detail in Cappellari et al. (2013b).

The same integral-field spectroscopy was used to measure the spectroscopic stellar mass-to-light ratio (assuming a Salpeter IMF), $(M / L)_{\text {Salp }}$. These were measured independently from the SSP parameters (which also assume a Salpeter IMF), using a regularized pPXF spectral fit of the MIUSCAT stellar population models from Vazdekis et al. (2012) as templates. To be consistent with the stellar kinematics used in the dynamical modeling, the spectra within an aperture extending to one effective radius were summed and used in the spectral fit. In all, 264 templates were simultaneously fitted to this aperture spectrum, giving the massweighted mean mass-to-light ratio for each galaxy taking into account a smooth, non-parametric star-formation history. The resulting $r$-band $(M / L)_{\text {stars }}$ and $(M / L)_{\text {Salp }}$ values are given in Table 1 of Cappellari et al. (2013a).

Using the same nomenclature as Cappellari et al. (2013a), we quantify the mass normalization of the IMF using the IMF parameter $\alpha_{\text {dyn }} \equiv(M / L)_{\text {stars }} /(M / L)_{\text {Salp }}$, such that $\alpha_{\text {dyn }}>1$ implies an IMF "heavier" than Salpeter. For the results that follow, we apply the same sample selection as that paper, ${ }^{23}$ excluding objects with $\mathrm{H} \beta>2.3 \AA$ within an effective radius, on the basis that they exhibit very strong age gradients, thus breaking the constant mass-to-light ratio assumption of the dynamical modeling approach, and making $(M / L)_{\text {Salp }}$ ill-defined. In addition, we exclude the object PGC071531, as the quality of the kinematic data was too poor to allow a secure measure of $\alpha_{\text {dyn }}$. The

\footnotetext{
23 This selection is tabulated in column (6) of the online version of Table 1 in Cappellari et al. (2013a).
}

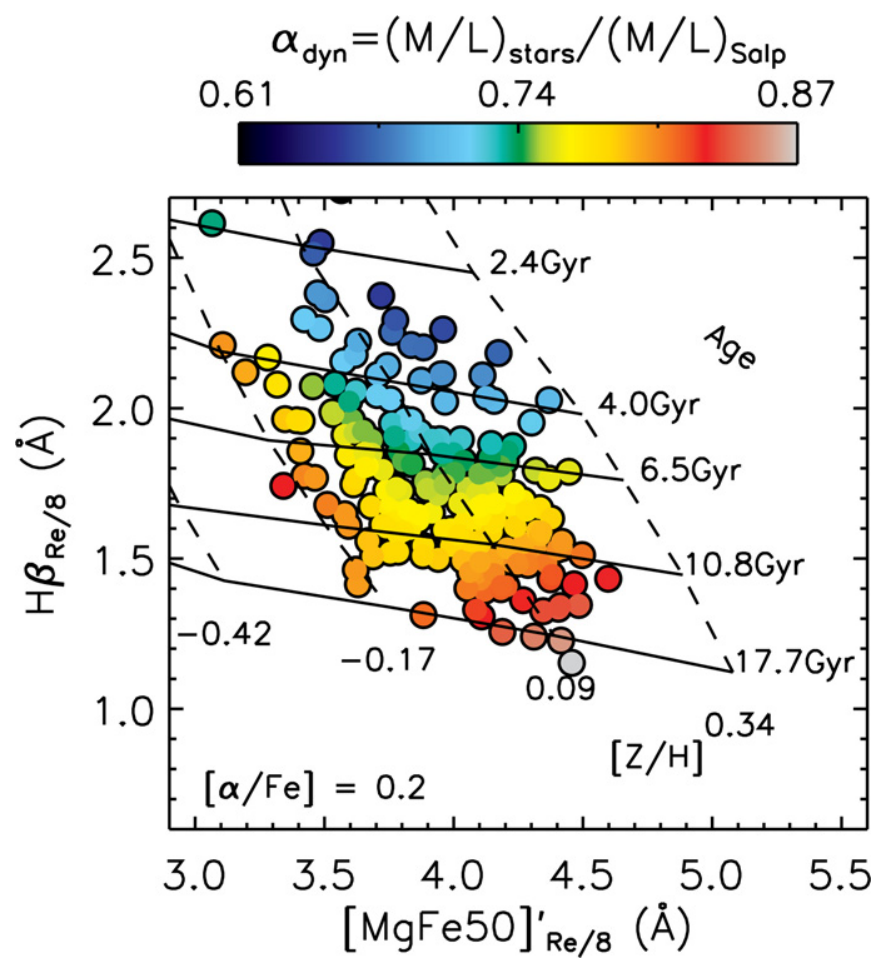

Figure 1. Lick indices $\mathrm{H} \beta$ vs. the combined index $[\mathrm{MgFe} 50]^{\prime}$, both measured within an aperture of one-eighth of an effective radius. The color scale indicates the value of the IMF parameter, $\alpha_{\text {dyn }}$, defined as the ratio of the mass-to-light ratios derived from dynamical modeling (accounting for dark matter) and stellar population modeling. The measured values of the IMF parameter have been adaptively smoothed using a locally weighted regression technique to show underlying trends in the distribution. Note that the range of values resulting from this averaging technique is necessarily reduced. A grid of SSP model predictions from Schiavon (2007) is shown for a super-solar abundance ratio of $[\alpha / \mathrm{Fe}]=0.2$ as indicated in the lower left of the plot. Solid lines indicate lines of constant age; dashed lines are constant metallicity, as shown.

(A color version of this figure is available in the online journal.)

following analysis uses the remaining 212 galaxies satisfying these criteria.

\section{RESULTS}

\subsection{IMF and Line-strengths}

We begin by showing the link between the IMF mass normalization (via $\alpha_{\text {dyn }}$ ) to simple empirical quantities to make our results independent of stellar population models. Figure 1 presents the distribution of the IMF parameter plotted in the twodimensional plane of line strength indices $\mathrm{H} \beta$ and $[\mathrm{MgFe} 50]^{\prime} .^{24}$ The former index is sensitive to stellar age; the latter is sensitive to metallicity in a way that is not strongly dependent on $[\alpha / \mathrm{Fe}]$ (Kuntschner et al. 2010). The values of the IMF parameter in this plane have been adaptively smoothed using the two-dimensional locally weighted regression robust technique (dubbed LOESS) of Cleveland \& Devlin (1988), as implemented in Cappellari et al. (2013a). ${ }^{25}$ The LOESS-smoothed distribution tries to remove observational errors and intrinsic scatter to estimate the mean values of the underlying galaxy population, thus approximating the mean values one would obtain from simple binning of much larger samples.

Overplotted on these points is a grid of SSP parameter predictions from Schiavon (2007) for a super-solar $[\alpha / \mathrm{Fe}]=0.2$.

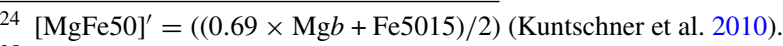

25 Available from purl.org/cappellari/software
} 

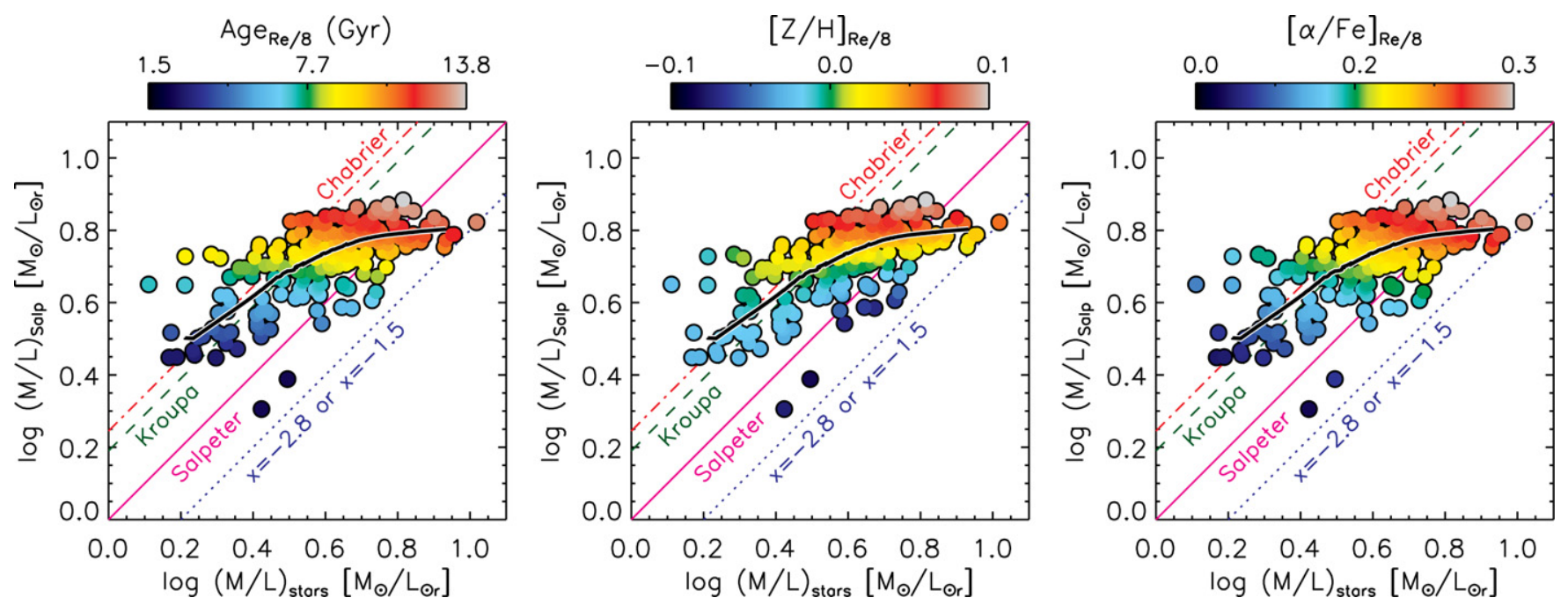

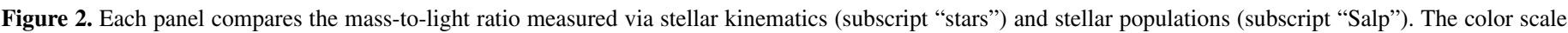

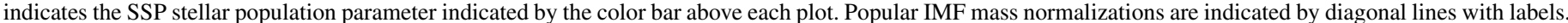
The thick black curve traces the ridge line of the points via a one-dimensional implementation of the LOESS locally weighted regression technique.

(A color version of this figure is available in the online journal.)

There is a trend of larger values of the IMF parameter (corresponding to "heavier" IMFs) toward older, metal-rich objects. The trend, however, is not monotonic, with lower metallicity objects also showing relatively high IMF parameter values on average.

The LOESS technique averages co-spatial data points and, as with any binning/averaging approach, the absolute range of values is reduced compared to the original data via reduction of intrinsic and measurement scatter (e.g., compare the color scale of Figure 1 with the intrinsic IMF values implied in Figure 2). The LOESS-averaged color-map plots are useful to not only uncover the underlying trends, but also quantitatively treat correlations of the actual values. In the following section we analyze the two-dimensional projections of IMF with stellar population parameters individually.

\subsection{IMF and SSP-parameters}

Each panel in Figure 2 presents our two mass-to-light ratios $(M / L)_{\text {stars }}$ and $(M / L)_{\text {Salp }}$ plotted against each other, overplotted with diagonal identity lines to indicate the corresponding IMF normalization, such that the IMF varies perpendicular to these diagonal lines. The thick black line shows the result of a onedimensional locally weighted regression (LOESS) analysis in order to trace the central ridge-line of the mass-to-light ratio points. The measured SSP parameters are indicated by colored points with the parameter name given in the plot title. Again, the SSP parameter values have been smoothed using the twodimensional LOESS algorithm used in Section 3.1 in order to show the average trend expected from a larger sample of galaxies. These plots are directly comparable to Figure 11 of Cappellari et al. (2013a) where the color scale was used to show how velocity dispersion varies in this plane.

It can be seen that the general trends agree with what was inferred above, namely that the IMF becomes systematically "heavier," on average, for galaxies that are older, more metalrich, and additionally, more enhanced in alpha elements. It is clear from these plots, however, that stellar population is not a good predictor of $\alpha_{\mathrm{dyn}}$. The average trend appears due to the fact that, while older, or alpha-enhanced, galaxies span a larger range of $\alpha_{\text {dyn }}$ values going from Kroupa to heavier than Salpeter, the range of $\alpha_{\text {dyn }}$ appears largely limited to Kroupa-like values for galaxies with young ages and low $[\alpha / \mathrm{Fe}]$. A similar conclusion can be drawn from Figure 11 of Cappellari et al. (2013a), where galaxies with low velocity dispersion are similarly limited to Kroupa-like IMF normalization. Metallicity shows a broadly similar trend, however, the detailed distribution is different from that with age and $[\alpha / \mathrm{Fe}]$, with the iso-metallicity bands running more parallel to the IMF normalization. Notably, there are objects with low metallicity that require an IMF heavier than Salpeter, as in Figure 1.

We note that the population parameters vary more tightly with $(M / L)_{\text {Salp }}$ than $(M / L)_{\text {stars }}$, reflecting the strong dependence of stellar population $M / L$ estimates on stellar population parameters. The dynamical $M / L$ estimate is independent of stellar evolutionary effects, which may explain the broader range of population parameters at fixed $(M / L)_{\text {stars }}$.

In Figure 3 we present the two-dimensional relations of the IMF parameter and population parameters, where points represent our individual measurements. A robust linear fit of the form $y=a+b x$ is made in each panel using the LTS_LINEFIT routine described in Cappellari et al. (2013b), which combines the Least Trimmed Squares robust technique of Rousseeuw \& Driessen (2006) into a least-squares fitting algorithm which allows for intrinsic scatter. Fit parameters are given in the upper-left of each panel. Our relations have around $25 \%$ scatter due to observational errors, with $12 \%$ intrinsic scatter. The Spearman's rank correlation coefficient $(r)$ and the two-sided significance of its deviation from zero $(0<p<1$, with smaller values implying higher significance) are given in the upper right. The correlation coefficient values, which are independent from the assumption of a linear relationship, confirm the presence of weak but statistically significant relationships of $\alpha_{\text {dyn }}$ with age and $[\alpha / \mathrm{Fe}]$ and no evidence of a correlation with total metallicity.

\section{DISCUSSION}

The main result of this Letter is that the trends between the dynamically derived IMF normalization parameter $\alpha_{\text {dyn }}$ and stellar population parameters are weak. We find that old or alphaenhanced galaxies have on average heavier IMF normalization, 

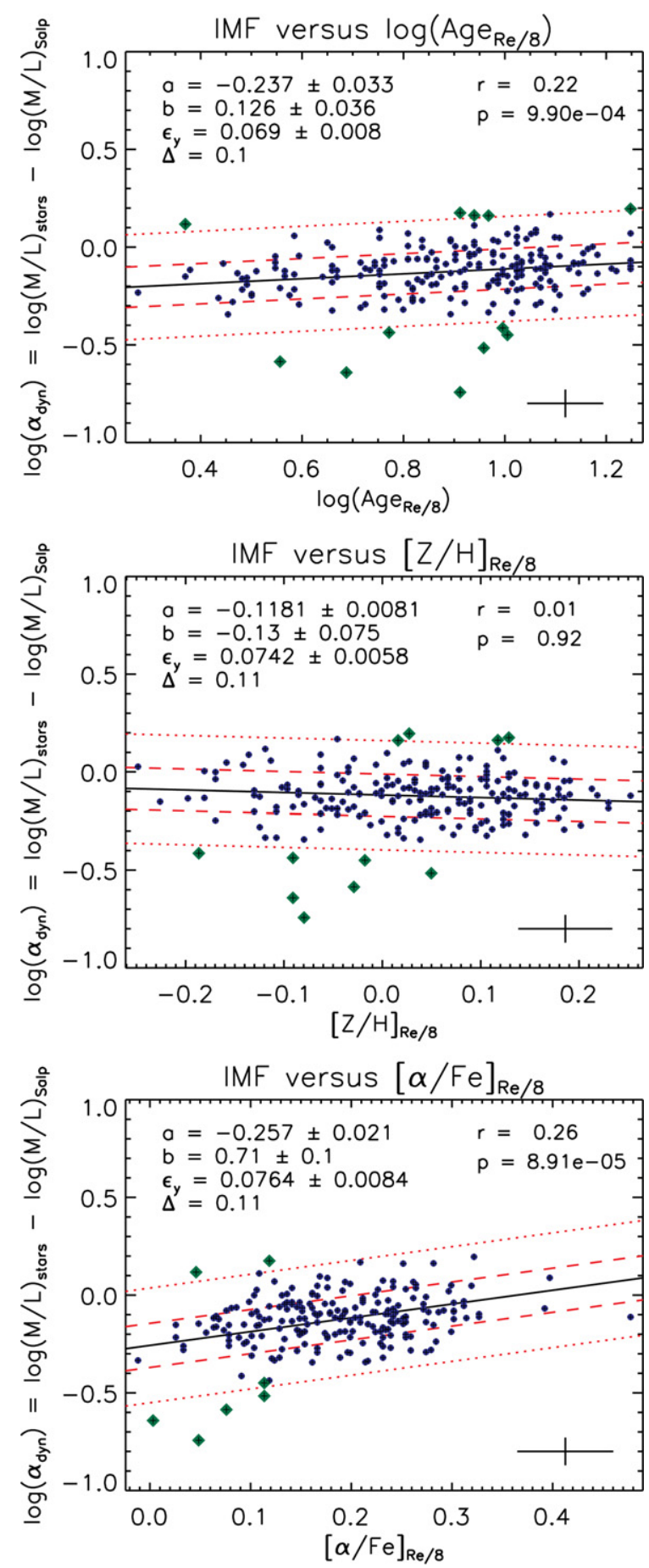

Figure 3. Relations between the IMF (vertical axis) and SSP parameters age (top left), metallicity (top right), and $[\alpha / \mathrm{Fe}]$ (bottom). A robust linear fit is shown (black solid line) together with $1 \sigma(68 \%)$ and $2.6 \sigma(99 \%)$ percentiles as red dashed and dotted lines, respectively. Blue circular symbols indicate points included in the fit, with green diamonds showing points rejected during the iterative fitting. Fit parameters are given in the upper left of each panel, giving intercept $(a)$, gradient $(b)$, intrinsic scatter in the $y$-direction $\left(\epsilon_{y}\right)$, and observed standard deviation around the fit $(\Delta)$. Individual errors were included in the fit, but for clarity, the median error for each panel is shown in the bottom right of each panel. Values in the upper right give the Spearman's rank correlation coefficient $(r)$ and significance $(p)$.

(A color version of this figure is available in the online journal.)

but a heavy IMF is not found in only those galaxies. Old (and high $[\alpha / \mathrm{Fe}])$ galaxies span a large range of IMF normalization going from Kroupa to Salpeter, while young (and low $[\alpha / \mathrm{Fe}]$ ) ones seem to almost exclusively have a Kroupa normalization.
Metallicity shows a less clear distinction, having a broad range of mass normalizations at all metallicity values (Figure 3, middle panel). Conroy \& van Dokkum (2012) also find a weaker relationship between IMF and total metallicity, $[\mathrm{Z} / \mathrm{H}]$, concluding, as here, that there is no compelling evidence of a correlation.

We have explored several avenues to verify the weak trends we find. For example, we find fully consistent results using the mass-weighted ages and metallicities from the spectral fits used to derive our $(M / L)_{\text {Salp }}$ instead of the SSP values, showing that the different models (Schiavon SSP models versus Vazdekis model spectra) and methods (chi-squared fitting for the best SSP versus pPXF spectral fitting of star formation histories for the mass-weighted values) yield consistent results. Using the spectrum integrated within a full effective radius $R_{e}$ to derive the SSP parameters (instead of $R_{e} / 8$ ) gives trends equivalent to Figure 3 within the $1 \sigma$ uncertainties, with comparable scatter, correlation coefficient values, and significance (using this larger aperture, the Spearman $(r, p)$ values become $\left(0.23,1.1 \times 10^{-3}\right)$, $(0.05,0.5)$, and $\left(0.22,2.1 \times 10^{-3}\right)$ for the equivalent relations of IMF with age, metallicity, and $[\alpha / \mathrm{Fe}]$, respectively). This reassures us that our choice of aperture is not important and that possible radial variations in the IMF (Pastorello et al. 2014) do not directly affect our conclusions.

It is well established that stellar population parameters correlate positively with velocity dispersion (Thomas et al. 2005; Graves et al. 2009; Thomas et al. 2010). Given the positive correlation between velocity dispersion and IMF presented in Cappellari et al. (2013a), it is tempting to conclude that the trends shown in Figure 3 are simply tracing this underlying trend of IMF with velocity dispersion, as argued in Smith (2014). However, the tightest correlation of our stellar population parameters with velocity dispersion is with total metallicity (McDermid et al. 2014). Conversely, metallicity shows no evidence for any direct, linear correlation with the IMF. This contrary trend of IMF with metallicity compared with age and $[\alpha / \mathrm{Fe}]$ suggests that our findings are not purely a result of the underlying correlation of IMF with velocity dispersion, as this would give rise to consistent behavior between all three stellar population parameters, which all positively correlate with velocity dispersion.

The weak trends we find here are consistent with the similarly weak trend between $\alpha_{\mathrm{dyn}}$ and velocity dispersion $(r=0.36, p=$ $5.7 \times 10^{-8}$ ), reported in Cappellari et al. (2013a). A comparison of the ATLAS ${ }^{3 \mathrm{D}}$ velocity dispersion-IMF relation with several published spectral studies (La Barbera et al. 2013; Conroy \& van Dokkum 2012; Treu et al. 2010; Spiniello et al. 2014) is given in Figure 12 of Spiniello et al. (2014). While there is overlap between the various spectral studies and ATLAS ${ }^{3 \mathrm{D}}$ over the range of velocity dispersion in common $\left(\sigma>130 \mathrm{~km} \mathrm{~s}^{-1}\right)$, the overall relationship for the entire sample is significantly more shallow than that suggested by the spectral studies. Restricting our sample to galaxies with this range of velocity dispersion (where the agreement is best) results in shallower gradients in the $\alpha_{\text {dyn }}-$ SSP relations presented here, with larger uncertainties (a zero gradient with age and $[\alpha / \mathrm{Fe}]$ is excluded with only $2 \sigma$ confidence rather than $5 \sigma)$, and smaller correlation coefficients $(r<0.16)$ with less significance $(p>0.02)$, consistent with no significant correlation of $\alpha_{\mathrm{dyn}}$ with any population parameter.

Other explanations for the weak trends are possible biases in the dynamical and/or population IMF estimates. An obvious potential systematic problem with the dynamically derived IMF, which has often been invoked in the past, is the treatment of the 
dark matter content in the dynamical models. We showed in Cappellari et al. (2012) that for dark matter to explain the IMF trends, it would have to follow the stellar distribution much closer than any current model predicts, making this explanation very unlikely.

Other sources of biases include general problems in the $(M / L)_{\text {Salp }}$ derived from stellar population models. Using the SSP models of Vazdekis et al. (2012) and Bruzual \& Charlot (2003) to derive $(M / L)_{\text {Salp }}$ from our line-strengths, we find that the weak age trend is removed due to the biased reduction in $(M / L)_{\text {Salp }}$ by young populations when using the (luminosityweighted) SSP approach instead of (mass-weighted) spectral fitting. Any relationship with $[\mathrm{Z} / \mathrm{H}]$ remains absent using this SSP approach, and the weak trend with $[\alpha / \mathrm{Fe}]$, though present, becomes less significant, with best-fit gradients excluding zero at only a $1 \sigma-4 \sigma$ level. Our weak trends are therefore a general outcome from different models and approaches.

Finally, we note that a direct comparison of the IMF parameters for galaxies in common between our study and Conroy \& van Dokkum (2012) yields only a weak relationship between the two studies (Smith 2014). The relatively small overlapping sample (34 objects), and differences in spatial apertures used in determining the IMF normalization (Conroy \& van Dokkum 2012 use a small central aperture, whereas our IMF analysis uses data from within, on average, one effective radius) preclude firm conclusions on how this lack of agreement relates to the systematic uncertainties of either study. Removing these remaining issues is the focus of future work.

\section{CONCLUSIONS}

We present the observed trends of stellar population parameters with the dynamically determined IMF for 212 early-type galaxies from the ATLAS ${ }^{3 \mathrm{D}}$ survey. Using adaptive smoothing to highlight the average trends comparable to studies of larger samples, we find that the IMF normalization tends on average to be "heavy" for both weak $\mathrm{H} \beta$ and/or weak metal absorption line strengths. Using SSP models, we show that the stellar population properties of age, metallicity, and $[\alpha / \mathrm{Fe}]$ span a broad range of values for any given IMF normalization.

We present the observed relations of IMF with the SSP properties of the individual galaxies of our sample. We find notably weak relations between the IMF and all three stellar population parameters, with mildly positive correlations of IMF "heaviness" with age and $[\alpha / \mathrm{Fe}]$, and no significant correlation with total metallicity. Taken together with the weak relation between the IMF normalization and velocity dispersion already presented in Cappellari et al. (2013a), these results are somewhat at variance with those from various recent spectroscopic studies. Future efforts for obtaining large samples of individual objects with integral-field spectroscopy that also provides IMF-sensitive spectral features are now critical to permit the responsible systematic uncertainties in local galaxy IMF studies to be resolved.

The authors thank the referee for helping to improve this article. The research leading to these results has received funding from the European Community's Seventh Framework Programme (/FP7/2007-2013/) under grant agreement
No. 229517. M.C. acknowledges support from a Royal Society University Research Fellowship. This work was supported by the rolling grants PP/E001114/1 and ST/H002456/1 and visitor's grants PPA/V/S/2002/00553, PP/E001564/1 and ST/H504862/1 from the UK Research Councils. R.L.D. acknowledges travel and computer grants from Christ Church, Oxford and support from the Royal Society in the form of a Wolfson Merit Award 502011.K502/jd. S.K. acknowledges support from the Royal Society Joint Projects Grant JP0869822. T.N. and M. Bois acknowledge support from the DFG Cluster of Excellence "Origin and Structure of the Universe." M.S. acknowledges support from a STFC Advanced Fellowship ST/F009186/1. During this research, M. Bois has received funding from the European Research Council under the Advanced Grant Program Num. 267399-Momentum. L.Y. acknowledges support from NSF grant AST-1109803. The authors acknowledge financial support from ESO.

\section{REFERENCES}

Auger, M. W., Treu, T., Bolton, A. S., et al. 2010, ApJ, 724, 511

Bastian, N., Covey, K. R., \& Meyer, M. R. 2010, ARA\&A, 48, 339

Bruzual, G., \& Charlot, S. 2003, MNRAS, 344, 1000

Cappellari, M. 2002, MNRAS, 333, 400

Cappellari, M. 2008, MNRAS, 390, 71

Cappellari, M., \& Emsellem, E. 2004, PASP, 116, 138

Cappellari, M., Emsellem, E., Krajnović, D., et al. 2011, MNRAS, 413, 813

Cappellari, M., McDermid, R. M., Alatalo, K., et al. 2012, Natur, 484, 485

Cappellari, M., McDermid, R. M., Alatalo, K., et al. 2013a, MNRAS, 432, 1862

Cappellari, M., Scott, N., Alatalo, K., et al. 2013b, MNRAS, 432, 1709

Cleveland, W., \& Devlin, S. 1988, Journal of the American Statistical Association, 83, 596

Conroy, C., Dutton, A. A., Graves, G. J., Mendel, J. T., \& van Dokkum, P. G. 2013, ApJL, 776, L26

Conroy, C., \& van Dokkum, P. 2012, ApJ, 760, 71

Emsellem, E., Monnet, G., \& Bacon, R. 1994, A\&A, 285, 723

Ferreras, I., La Barbera, F., de la Rosa, I. G., et al. 2013, MNRAS, 429, L15

Graves, G. J., Faber, S. M., \& Schiavon, R. P. 2009, ApJ, 698, 1590

Kroupa, P. 2002, Sci, 295, 82

Kuntschner, H., Emsellem, E., Bacon, R., et al. 2010, MNRAS, 408, 97

La Barbera, F., Ferreras, I., Vazdekis, A., et al. 2013, MNRAS, 433, 3017

McDermid, R. M., Alatalo, K., Blitz, L., et al. 2014, MNRAS, submitted

McDermid, R. M., Emsellem, E., Shapiro, K. L., et al. 2006, MNRAS, 373, 906

Navarro, J. F., Frenk, C. S., \& White, S. D. M. 1996, ApJ, 462, 563

Pastorello, N., Forbes, D. A., Foster, C., et al. 2014, MNRAS, 442, 1003

Peacock, M. B., Zepf, S. E., Maccarone, T. J., et al. 2014, ApJ, 784, 162

Rousseeuw, P. J., \& Driessen, K. 2006, Data Mining and Knowledge Discovery, 12,1

Salpeter, E. E. 1955, ApJ, 121, 161

Schiavon, R. P. 2007, ApJS, 171, 146

Scott, N., Cappellari, M., Davies, R. L., et al. 2013, MNRAS, 432, 1894

Smith, R. J. 2014, MNRAS, 443, L69

Smith, R. J., \& Lucey, J. R. 2013, MNRAS, 434, 1964

Smith, R. J., Lucey, J. R., \& Carter, D. 2012, MNRAS, 426, 2994

Spiniello, C., Trager, S. C., Koopmans, L. V. E., \& Chen, Y. P. 2012, ApJL, 753, L32

Spiniello, C., Trager, S., Koopmans, L. V. E., \& Conroy, C. 2014, MNRAS, 438, 1483

Thomas, D., Maraston, C., Bender, R., \& Mendes de Oliveira, C. 2005, ApJ, 621,673

Thomas, D., Maraston, C., Schawinski, K., Sarzi, M., \& Silk, J. 2010, MNRAS, 404, 1775

Tortora, C., Romanowsky, A. J., \& Napolitano, N. R. 2013, ApJ, 765, 8

Treu, T., Auger, M. W., Koopmans, L. V. E., et al. 2010, ApJ, 709, 1195

van Dokkum, P. G., \& Conroy, C. 2010, Natur, 468, 940

van Dokkum, P. G., \& Conroy, C. 2011, ApJL, 735, L13

Vazdekis, A., Ricciardelli, E., Cenarro, A. J., et al. 2012, MNRAS, 424, 157

Worthey, G., \& Ottaviani, D. L. 1997, ApJS, 111, 377 